Supplement of Atmos. Chem. Phys., 19, 5403-5415, 2019

https://doi.org/10.5194/acp-19-5403-2019-supplement

(c) Author(s) 2019. This work is distributed under

the Creative Commons Attribution 4.0 License.

(c) (1)

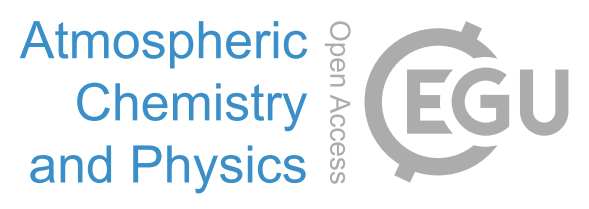

Supplement of

\title{
Simulation of the chemical evolution of biomass burning organic aerosol
}

\section{Georgia N. Theodoritsi and Spyros N. Pandis}

Correspondence to: Spyros Pandis (spyros@chemeng.upatras.gr)

The copyright of individual parts of the supplement might differ from the CC BY 4.0 License. 
1 Table S1. POA and bbPOA emission rates (in tn $\mathrm{d}^{-1}$ ) for each volatility bin during the 2 simulated periods.

\begin{tabular}{llllllllll}
\hline $\begin{array}{l}\mathrm{C}^{*} \text { at } 298 \mathrm{~K} \\
\left(\mu \mathrm{g} \mathrm{m}^{-3}\right)\end{array}$ & $10^{-2}$ & $10^{-1}$ & $10^{0}$ & $10^{1}$ & $10^{2}$ & $10^{3}$ & $10^{4}$ & $10^{5}$ & $10^{6}$ \\
\hline
\end{tabular}

1-29 May 2008 emission rate $\left(\operatorname{tn~}^{-1}\right)$

\begin{tabular}{|c|c|c|c|c|c|c|c|c|c|}
\hline fPOA & 100 & 200 & 310 & 480 & 620 & 1000 & 1400 & 1700 & 2700 \\
\hline $\begin{array}{l}\text { bbPOA (Base } \\
\text { case) }\end{array}$ & 530 & 0 & 260 & 260 & 530 & 260 & 800 & 1300 & 2100 \\
\hline $\begin{array}{l}\text { bbPOA } \\
\text { (Sensitivity } \\
\text { test) }\end{array}$ & 580 & 0 & 290 & 290 & 580 & 290 & 870 & - & - \\
\hline \multicolumn{10}{|c|}{25 February - 22 March 2009 emission rate $\left(\operatorname{tn~d~}^{-1}\right)$} \\
\hline fPOA & 70 & 140 & 210 & 330 & 420 & 700 & 930 & 1200 & 1900 \\
\hline $\begin{array}{l}\text { bbPOA (Base } \\
\text { case) }\end{array}$ & 2100 & 0 & 1000 & 1000 & 2100 & 1000 & 3100 & 5100 & 8200 \\
\hline $\begin{array}{l}\text { bbPOA } \\
\text { (Sensitivity } \\
\text { test) }\end{array}$ & 2300 & 0 & 1100 & 1100 & 2300 & 1100 & 3400 & - & - \\
\hline
\end{tabular}


1 Table S2. Prediction skill metrics of PMCAMx-SR (base case) against AMS factor 2 analysis in May $2008^{\mathrm{a}}$.

\begin{tabular}{ccccccc}
\hline & $\begin{array}{c}\text { Mean } \\
\text { Observed } \\
\left(\mu \mathrm{g} \mathrm{m}^{-3}\right)\end{array}$ & $\begin{array}{c}\text { Mean } \\
\text { Predicted } \\
\left(\mu \mathrm{g} \mathrm{m}^{-3}\right)\end{array}$ & $\begin{array}{c}\text { MB } \\
\left(\mu \mathrm{g} \mathrm{m}^{-3}\right)\end{array}$ & $\begin{array}{c}\text { MAGE } \\
\left(\mu \mathrm{g} \mathrm{m}^{-3}\right)\end{array}$ & FBIAS & FERROR \\
\hline CA & 4.37 & 5.55 & 1.18 & 1.68 & 0.30 & 0.39 \\
HOA & 0.59 & 0.33 & -0.27 & 0.31 & -0.56 & 0.69 \\
BBOA & 0.43 & 0.42 & -0.01 & 0.36 & -0.18 & 0.53 \\
OOA & 3.34 & 4.80 & 1.46 & 1.64 & 0.42 & 0.46 \\
\hline \multicolumn{7}{c}{ Finokalia } \\
\hline OA & 2.49 & 3.00 & 0.50 & 0.92 & 0.21 & 0.36 \\
HOA & 0.09 & 0.03 & -0.06 & 0.07 & -0.81 & 0.97 \\
BBOA & - & 0.03 & - & - & - & - \\
OOA & 2.41 & 2.94 & 0.54 & 0.92 & 0.23 & 0.37 \\
\hline \multicolumn{7}{c}{ Melpitz } \\
\hline OA & 5.12 & 4.45 & -0.68 & 1.32 \\
HOA & 0.27 & 0.14 & -0.13 & 0.17 & -0.13 & 0.28 \\
BBOA & - & 0.12 & - & - & - & 0.83 \\
OOA & 4.86 & 4.19 & -0.66 & 1.29 & -0.14 & 0.29 \\
\hline \multicolumn{7}{c}{ Mace Head } \\
\hline OA & 2.29 & 2.62 & 0.34 & 1.08 \\
HOA & 0.28 & 0.07 & -0.21 & 0.21 & -1.09 & 1.12 \\
BBOA & 0.39 & 0.04 & -0.35 & 0.35 & -1.66 & 1.66 \\
OOA & 1.61 & 2.51 & 0.89 & 1.12 & 0.34 & 0.50 \\
\hline
\end{tabular}

3 aNumber of data points; Cabauw: 623; Finokalia: 494; Melpitz: 155; Mace Head: 329. 
1 Table S3. Prediction skill metrics of PMCAMx-SR (sensitivity test) against AMS

2 factor analysis in May $2008^{\mathrm{a}}$.

\begin{tabular}{|c|c|c|c|c|c|c|}
\hline & $\begin{array}{c}\text { Mean } \\
\text { Observed } \\
\left(\mu \mathrm{g} \mathrm{m}^{-3}\right)\end{array}$ & $\begin{array}{c}\text { Mean } \\
\text { Predicted } \\
\left(\mu \mathrm{g} \mathrm{m}^{-3}\right)\end{array}$ & $\begin{array}{c}\mathrm{MB} \\
\left(\mu \mathrm{g} \mathrm{m}^{-3}\right)\end{array}$ & $\begin{array}{l}\text { MAGE } \\
\left(\mu \mathrm{g} \mathrm{m}^{-3}\right)\end{array}$ & FBIAS & FERROR \\
\hline & \multicolumn{6}{|c|}{ Cabauw } \\
\hline $\mathrm{OA}$ & 4.37 & 5.23 & 0.86 & 1.49 & 0.25 & 0.36 \\
\hline HOA & 0.59 & 0.33 & -0.27 & 0.32 & -0.57 & 0.71 \\
\hline $\mathrm{BBOA}$ & 0.43 & 0.46 & 0.03 & 0.38 & -0.14 & 0.54 \\
\hline \multirow[t]{2}{*}{ OOA } & 3.34 & 4.44 & 1.10 & 1.35 & 0.35 & 0.41 \\
\hline & \multicolumn{6}{|c|}{ Finokalia } \\
\hline $\mathrm{OA}$ & 2.45 & 2.72 & 0.22 & 0.79 & 0.12 & 0.33 \\
\hline HOA & 0.09 & 0.03 & -0.07 & 0.07 & -0.83 & 0.99 \\
\hline $\mathrm{BBOA}$ & - & 0.03 & - & - & - & - \\
\hline OOA & 2.41 & 2.65 & 0.25 & 0.77 & 0.14 & 0.33 \\
\hline \multicolumn{7}{|c|}{ Melpitz } \\
\hline $\mathrm{OA}$ & 5.12 & 4.16 & -0.96 & 1.38 & -0.19 & 0.29 \\
\hline HOA & 0.27 & 0.13 & -0.13 & 0.17 & -0.63 & 0.84 \\
\hline BBOA & - & 0.13 & - & - & - & - \\
\hline OOA & 4.86 & 3.89 & -0.96 & 1.34 & -0.21 & 0.31 \\
\hline \multicolumn{7}{|c|}{ Mace Head } \\
\hline $\mathrm{OA}$ & 2.29 & 2.49 & 0.20 & 0.99 & 0.01 & 0.42 \\
\hline HOA & 0.28 & 0.07 & -0.21 & 0.21 & -1.11 & 1.13 \\
\hline $\mathrm{BBOA}$ & 0.39 & 0.05 & -0.34 & 0.34 & -1.63 & 1.63 \\
\hline OOA & 1.61 & 2.37 & 0.76 & 1.01 & 0.29 & 0.48 \\
\hline
\end{tabular}

$3 \quad$ anumber of data points; Cabauw: 623; Finokalia: 494; Melpitz: 155; Mace Head: 329. 
1 Table S4. Prediction skill metrics of PMCAMx-SR (base case) against AMS factor 2 analysis during 25 February - 23 March 2009a.

\begin{tabular}{|c|c|c|c|c|c|c|}
\hline & $\begin{array}{c}\text { Mean } \\
\text { Observed } \\
\left(\mu \mathrm{g} \mathrm{m}^{-3}\right)\end{array}$ & $\begin{array}{c}\text { Mean } \\
\text { Predicted } \\
\left(\mu \mathrm{g} \mathrm{m}^{-3}\right)\end{array}$ & $\begin{array}{c}\mathrm{MB} \\
\left(\mu \mathrm{g} \mathrm{m}^{-3}\right)\end{array}$ & $\begin{array}{l}\text { MAGE } \\
\left(\mu \mathrm{g} \mathrm{m}^{-3}\right)\end{array}$ & FBIAS & FERROR \\
\hline \multicolumn{7}{|c|}{ Cabauw } \\
\hline $\mathrm{OA}$ & 1.34 & 2.53 & 1.19 & 1.50 & 0.58 & 0.79 \\
\hline HOA & 0.25 & 0.55 & 0.30 & 0.38 & 0.71 & 0.90 \\
\hline BBOA & 0.13 & 1.05 & 0.93 & 0.94 & 1.35 & 1.40 \\
\hline OOA & 0.96 & 0.93 & -0.03 & 0.53 & 0.11 & 0.68 \\
\hline \multicolumn{7}{|c|}{ Helsinki } \\
\hline OA & 2.84 & 3.85 & 1.01 & 1.28 & 0.37 & 0.43 \\
\hline HOA & 0.45 & 0.29 & -0.16 & 0.25 & -0.14 & 0.66 \\
\hline BBOA & 0.40 & 1.67 & 1.27 & 1.27 & 1.19 & 1.19 \\
\hline OOA & 1.99 & 1.89 & -0.09 & 0.58 & -0.02 & 0.37 \\
\hline \multicolumn{7}{|c|}{ Mace Head } \\
\hline $\mathrm{OA}$ & 0.95 & 0.64 & -0.30 & 1.33 & 0.33 & 1.38 \\
\hline HOA & 0.09 & 0.004 & -0.09 & 0.09 & -0.53 & 1.15 \\
\hline BBOA & 0.34 & 0.01 & -0.32 & 0.34 & -0.42 & 1.24 \\
\hline OOA & 0.61 & 0.63 & 0.02 & 0.99 & 0.38 & 1.37 \\
\hline \multicolumn{7}{|c|}{ Melpitz } \\
\hline $\mathrm{OA}$ & 1.62 & 1.23 & -0.39 & 0.94 & -0.26 & 0.71 \\
\hline HOA & 0.15 & 0.09 & -0.05 & 0.08 & -0.31 & 0.68 \\
\hline BBOA & 0.18 & 0.37 & 0.19 & 0.28 & 0.69 & 0.93 \\
\hline OOA & 1.29 & 0.76 & -0.52 & 0.85 & -0.49 & 0.88 \\
\hline \multicolumn{7}{|c|}{ Hyytiala } \\
\hline $\mathrm{OA}$ & 1.45 & 2.36 & 0.92 & 1.12 & 0.55 & 0.66 \\
\hline $\mathrm{HOA}$ & 0.05 & 0.14 & 0.09 & 0.09 & 0.83 & 0.93 \\
\hline BBOA & 0.07 & 0.82 & 0.75 & 0.75 & 1.66 & 1.66 \\
\hline OOA & 1.33 & 1.41 & 0.08 & 0.69 & 0.12 & 0.58 \\
\hline \multicolumn{7}{|c|}{ Barcelona } \\
\hline $\mathrm{OA}$ & 8.66 & 3.62 & -5.04 & 5.31 & -0.68 & 0.78 \\
\hline HOA & 3.53 & 0.21 & -3.32 & 3.32 & -1.62 & 1.63 \\
\hline BBOA & 0.69 & 1.09 & 0.41 & 0.72 & 0.46 & 0.80 \\
\hline OOA & 4.45 & 2.32 & -2.13 & 2.42 & -0.51 & 0.69 \\
\hline \multicolumn{7}{|c|}{ Chilbolton } \\
\hline $\mathrm{OA}$ & 2.49 & 1.12 & -1.39 & 1.54 & -0.61 & 0.81 \\
\hline $\mathrm{HOA}$ & 0.50 & 0.19 & -0.32 & 0.33 & -0.88 & 0.93 \\
\hline BBOA & 0.49 & 0.24 & -0.26 & 0.36 & -0.72 & 1.07 \\
\hline OOA & 1.49 & 0.69 & -0.79 & 0.99 & -0.55 & 0.87 \\
\hline
\end{tabular}

$3 \quad{ }^{a}$ Number of data points; Cabauw: 418; Helsinki: 350; Mace Head: 206; Melpitz: 314; 4 Hyytiala: 426; Barcelona: 485; Chilbolton: 491. 
1 Table S5. Prediction skill metrics of PMCAMx-SR (sensitivity test) against AMS

2 factor analysis during 25 February - 23 March 2009ª

\begin{tabular}{|c|c|c|c|c|c|c|}
\hline & $\begin{array}{c}\text { Mean } \\
\text { Observed } \\
\left(\mu \mathrm{g} \mathrm{m}^{-3}\right)\end{array}$ & $\begin{array}{c}\text { Mean } \\
\text { Predicted } \\
\left(\mu \mathrm{g} \mathrm{m}^{-3}\right)\end{array}$ & $\begin{array}{c}\mathrm{MB} \\
\left(\mu \mathrm{g} \mathrm{m}^{-3}\right)\end{array}$ & $\begin{array}{l}\text { MAGE } \\
\left(\mu \mathrm{g} \mathrm{m}^{-3}\right)\end{array}$ & FBIAS & FERROR \\
\hline \multicolumn{7}{|c|}{ Cabauw } \\
\hline OA & 1.34 & 2.65 & 1.31 & 1.59 & 0.60 & 0.81 \\
\hline $\mathrm{HOA}$ & 0.25 & 0.55 & 0.31 & 0.38 & 0.72 & 0.91 \\
\hline BBOA & 0.13 & 1.16 & 1.04 & 1.05 & 1.39 & 1.44 \\
\hline OOA & 0.96 & 0.93 & -0.03 & 0.52 & 0.11 & 0.68 \\
\hline \multicolumn{7}{|c|}{ Helsinki } \\
\hline OA & 2.84 & 3.98 & 1.14 & 1.39 & 0.39 & 0.45 \\
\hline $\mathrm{HOA}$ & 0.45 & 0.28 & -0.16 & 0.25 & -0.14 & 0.66 \\
\hline BBOA & 0.40 & 1.84 & 1.44 & 1.44 & 1.24 & 1.24 \\
\hline OOA & 1.99 & 1.86 & -0.14 & 0.58 & -0.04 & 0.36 \\
\hline \multicolumn{7}{|c|}{ Mace Head } \\
\hline $\mathrm{OA}$ & 1.04 & 0.63 & -0.41 & 1.41 & 0.31 & 1.38 \\
\hline $\mathrm{HOA}$ & 0.09 & 0.004 & -0.09 & 0.09 & -0.53 & 1.15 \\
\hline BBOA & 0.34 & 0.01 & -0.32 & 0.34 & -0.38 & 1.24 \\
\hline OOA & 0.61 & 0.62 & 0.004 & 0.98 & 0.38 & 1.37 \\
\hline \multicolumn{7}{|c|}{ Melpitz } \\
\hline OA & 1.62 & 1.25 & -0.36 & 0.94 & -0.25 & 0.70 \\
\hline $\mathrm{HOA}$ & 0.15 & 0.09 & -0.05 & 0.08 & -0.31 & 0.68 \\
\hline BBOA & 0.18 & 0.41 & 0.23 & 0.31 & 0.76 & 0.96 \\
\hline OOA & 1.29 & 0.75 & -0.54 & 0.85 & -0.51 & 0.88 \\
\hline \multicolumn{7}{|c|}{ Hyytiala } \\
\hline OA & 1.45 & 2.41 & 0.96 & 1.14 & 0.57 & 0.66 \\
\hline $\mathrm{HOA}$ & 0.05 & 0.14 & 0.08 & 0.09 & 0.82 & 0.93 \\
\hline BBOA & 0.07 & 0.89 & 0.83 & 0.83 & 1.68 & 1.68 \\
\hline OOA & 1.33 & 1.37 & 0.04 & 0.67 & 0.11 & 0.57 \\
\hline \multicolumn{7}{|c|}{ Barcelona } \\
\hline OA & 8.66 & 3.52 & -5.15 & 5.39 & -0.69 & 0.79 \\
\hline HOA & 3.53 & 0.21 & -3.32 & 3.32 & -1.62 & 1.63 \\
\hline BBOA & 0.69 & 1.19 & 0.51 & 0.79 & 0.52 & 0.83 \\
\hline OOA & 4.45 & 2.11 & -2.34 & 2.56 & -0.56 & 0.74 \\
\hline \multicolumn{7}{|c|}{ Chilbolton } \\
\hline $\mathrm{OA}$ & 2.49 & 1.14 & -1.35 & 1.53 & -0.60 & 0.81 \\
\hline $\mathrm{HOA}$ & 0.50 & 0.19 & -0.32 & 0.32 & -0.87 & 0.93 \\
\hline BBOA & 0.49 & 0.26 & -0.24 & 0.35 & -0.66 & 1.03 \\
\hline OOA & 1.49 & 0.69 & -0.79 & 0.99 & -0.56 & 0.87 \\
\hline
\end{tabular}

$3 \quad{ }^{a}$ Number of data points; Cabauw: 418; Helsinki: 350; Mace Head: 206; Melpitz: 314;

4 Hyytiala: 426; Barcelona: 485; Chilbolton: 491. 
1 Table S6. Prediction skill metrics of PMCAMx and PMCAMx-SR against AMS 2 hourly measurements during May $2008^{\mathrm{a}}$.

3

\begin{tabular}{ccccccc}
\hline & $\begin{array}{c}\text { Mean } \\
\text { Observed } \\
\left(\mu \mathrm{g} \mathrm{m}^{-3}\right)\end{array}$ & $\begin{array}{c}\text { Mean } \\
\text { Predicted } \\
\left(\mu \mathrm{g} \mathrm{m}^{-3}\right)\end{array}$ & $\begin{array}{c}\text { MB } \\
\left(\mu \mathrm{g} \mathrm{m}^{-3}\right)\end{array}$ & $\begin{array}{c}\text { MAGE } \\
\left(\mu \mathrm{g} \mathrm{m}^{-3}\right)\end{array}$ & FBIAS & FERROR \\
\hline \multicolumn{7}{c}{ PMCAMx } \\
\hline OA & 3.43 & 4.01 & 0.57 & 1.26 & 0.17 & 0.37 \\
OOA & 2.84 & 3.71 & 0.87 & 1.29 & 0.29 & 0.42 \\
\hline \multicolumn{7}{c}{ PMCAMx-SR (Base Case) } \\
\hline OA & 3.44 & 4.06 & 0.62 & 1.29 & 0.18 & 0.38 \\
OOA & 2.84 & 3.70 & 0.85 & 1.28 & 0.29 & 0.42 \\
\hline OA & 3.44 & 3.79 & 0.35 & 1.16 & 0.12 & 0.36 \\
OOA & 2.84 & 3.41 & 0.57 & 1.10 & 0.22 & 0.39 \\
\hline \multicolumn{7}{c}{ PMCAMx-SR (Sensitivity test) }
\end{tabular}

4

${ }^{\text {a }}$ Sites: Cabauw, Finokalia, Melpitz and Mace Head. Number of data points: 1601.

6

7

8

9

10

11

12

13

14

15

16

17

18

19

20

21

22 
1 Table S7. Prediction skill metrics of PMCAMx and PMCAMx-SR against AMS

2 hourly measurements during 25 February - 23 March 2009a.

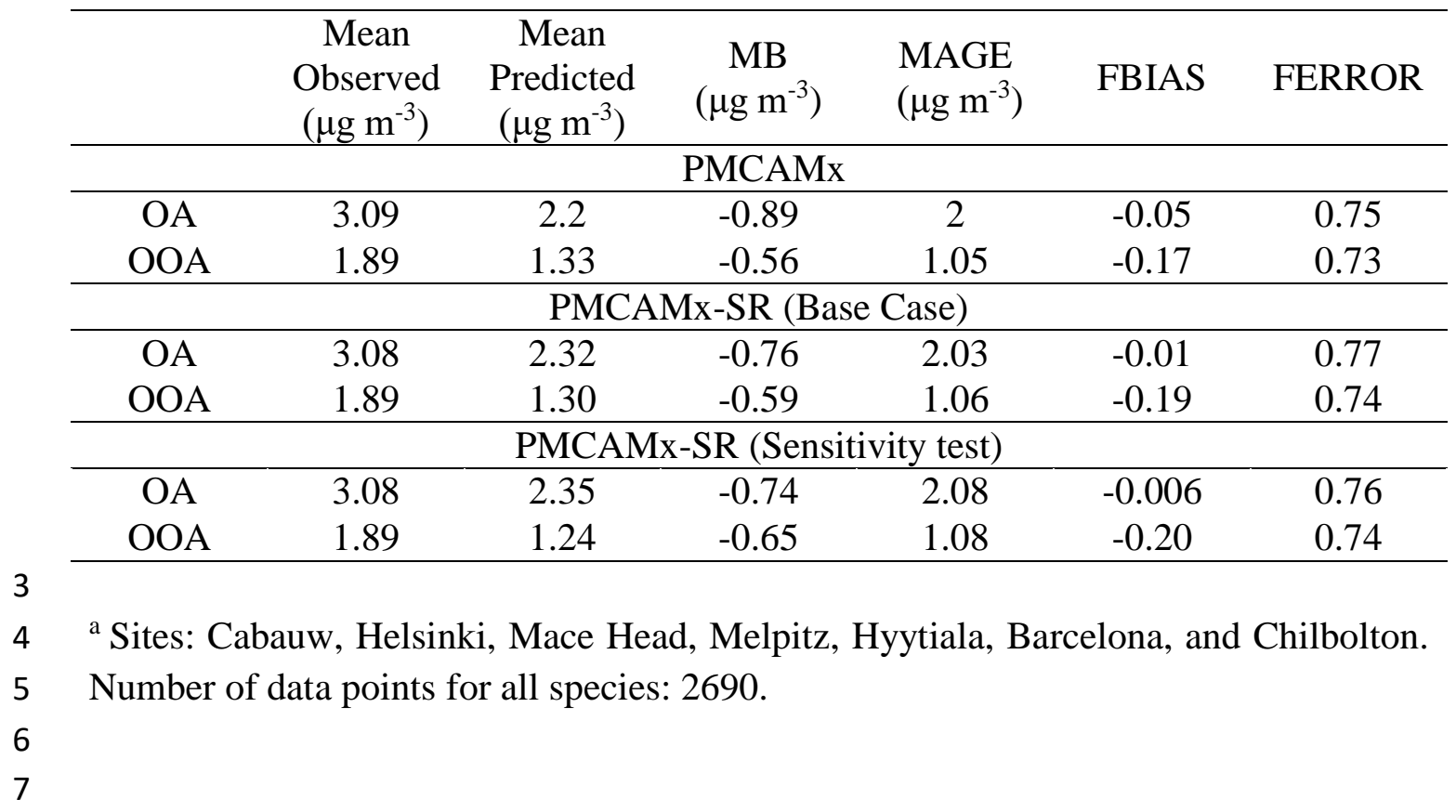




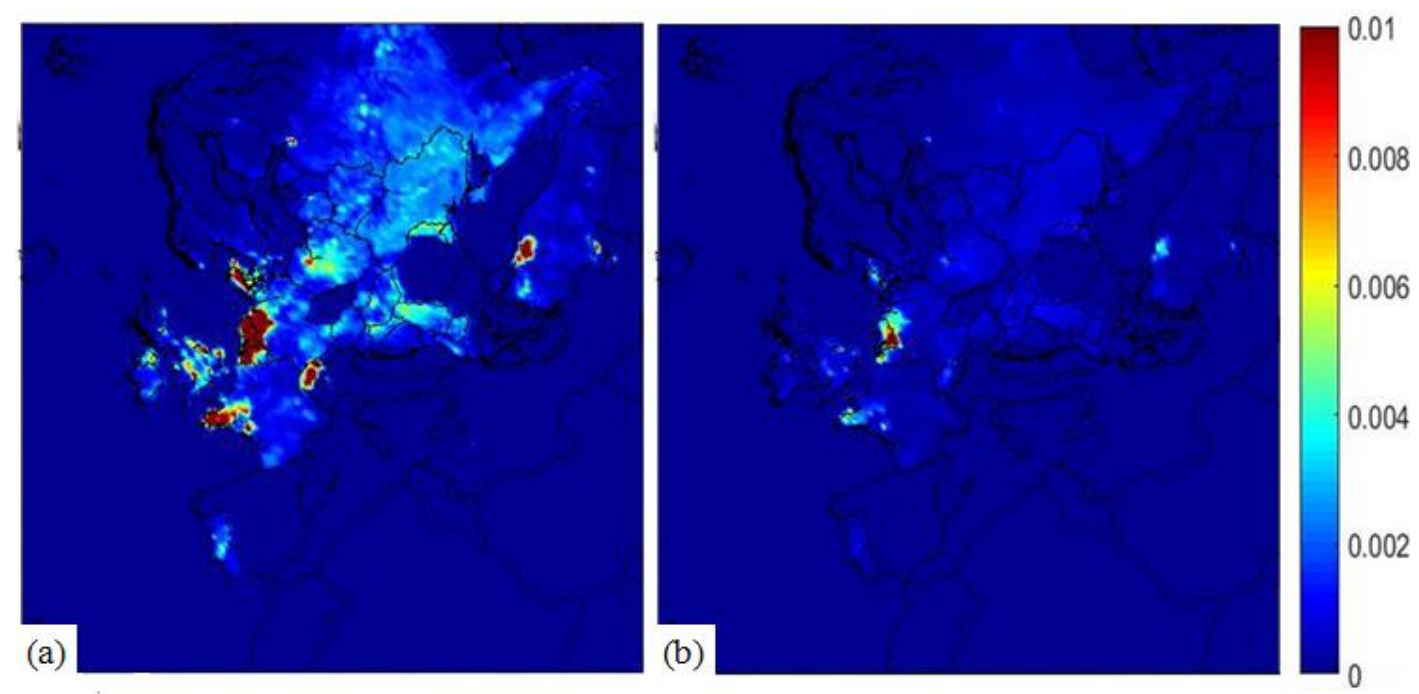

2 Figure S1. OA ground level emission rates $\left(\mathrm{kg} \mathrm{d}^{-1} \mathrm{~km}^{-2}\right)$ spatial distribution from 3 agricultural activities (waste burning) for (a) 1-29 May 2008 and (b) 25 February-22 4 March 2009. During both periods the average value of this source is approximately 7 $5 \times 10^{-3} \mathrm{~kg} \mathrm{~d}^{-1} \mathrm{~km}^{-2}$ and the maximum value is nearly $4 \mathrm{~kg} \mathrm{~d}^{-1} \mathrm{~km}^{-2}$ in the Netherlands. 


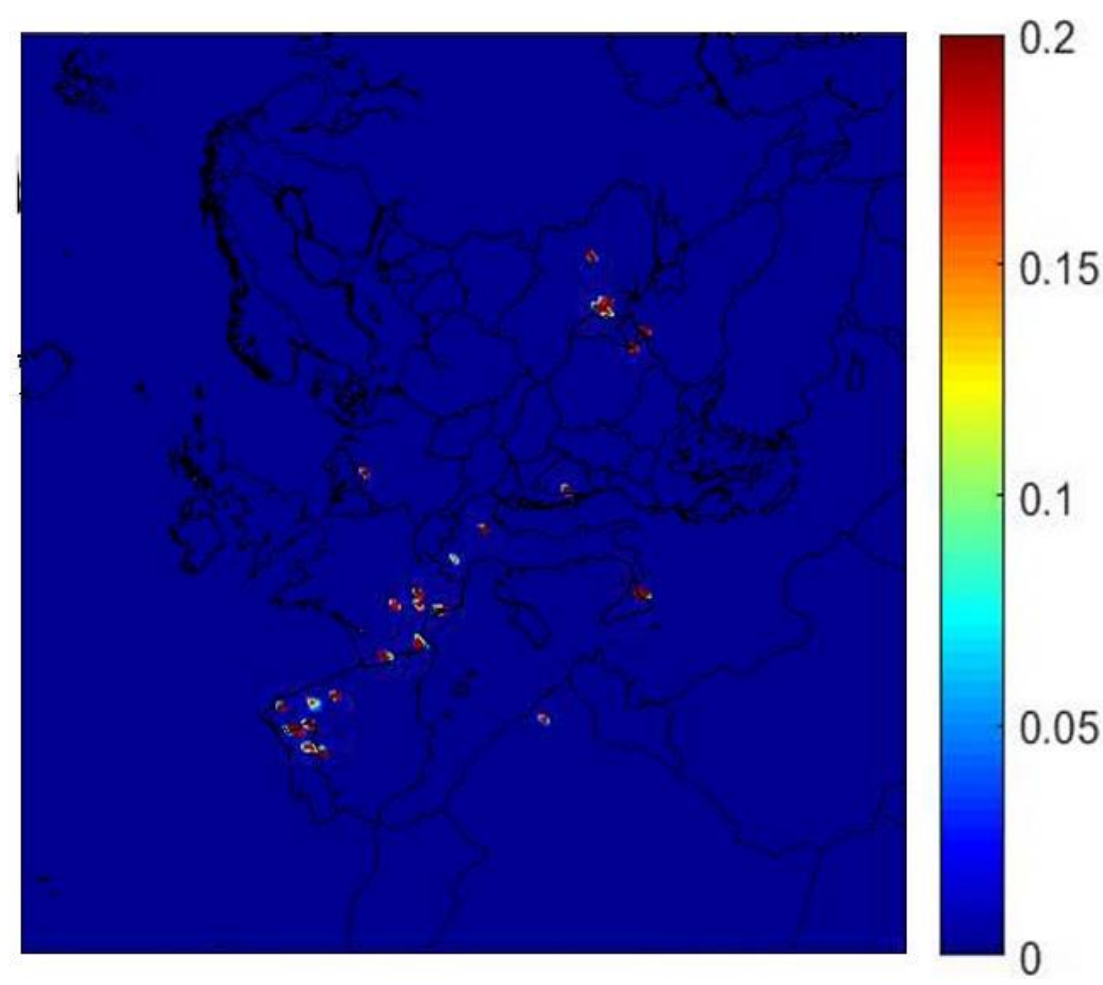

2 Figure S2. OA ground level emission rates $\left(\mathrm{kg} \mathrm{d}^{-1} \mathrm{~km}^{-2}\right)$ spatial distribution from 3 wildfires during 25 February-22 March 2009. Nearly 2000 tn day $^{-1}$ are emitted in 4 northwestern Spain and northern Portugal. The 2009 national fire data from these two 5 countries based on the European Forest Fire Information System (EFFIS) 6 (http://effis.jrc.ec.europa.eu) indicated intense fire activity in the second half of March 7 driven by dry weather and strong winds.

8 
Majden (FYROM)

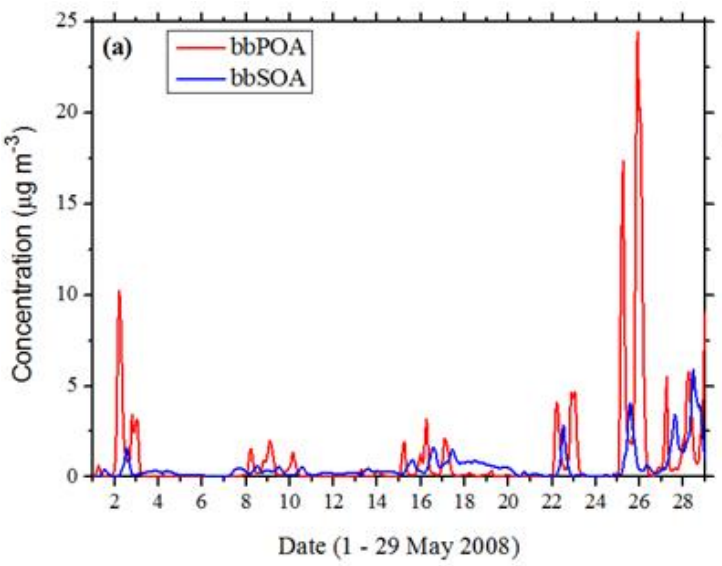

3

4

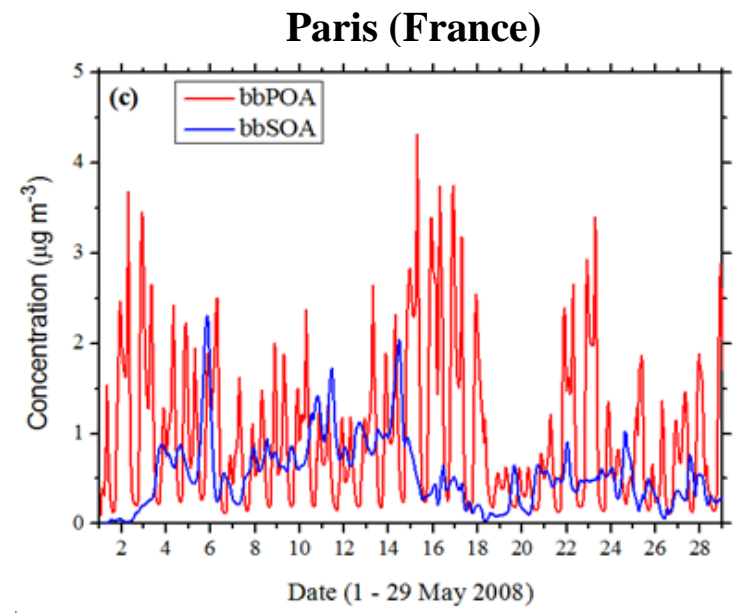

Catania (Italy)

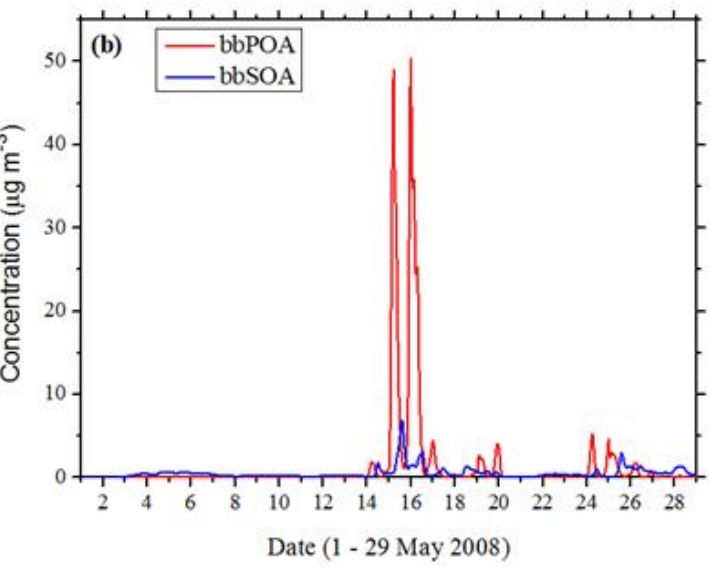

Dusseldorf (Germany)

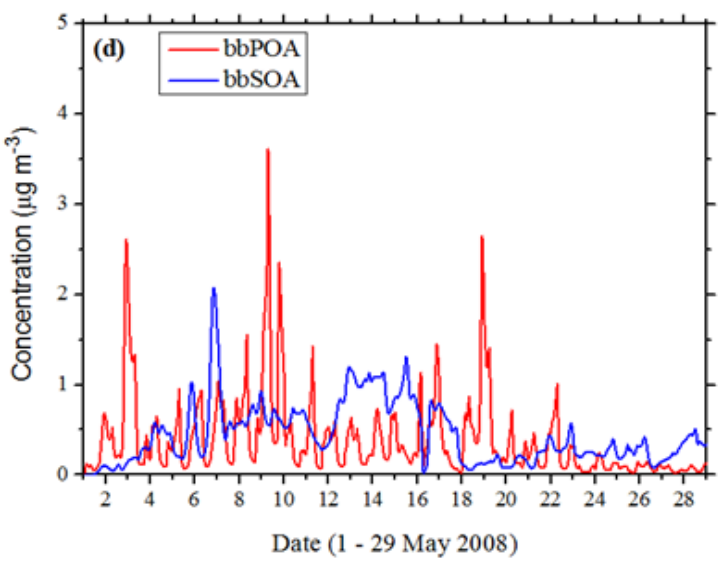

6

7 Figure S3. Timeseries of $\mathrm{PM}_{2.5}$ bbOA concentrations in (a) Majden (FYROM), (b)

8 Catania (Italy), (c) Paris (France) and (d) Dusseldorf (Germany) during 1-29 May 92008. 
Summer
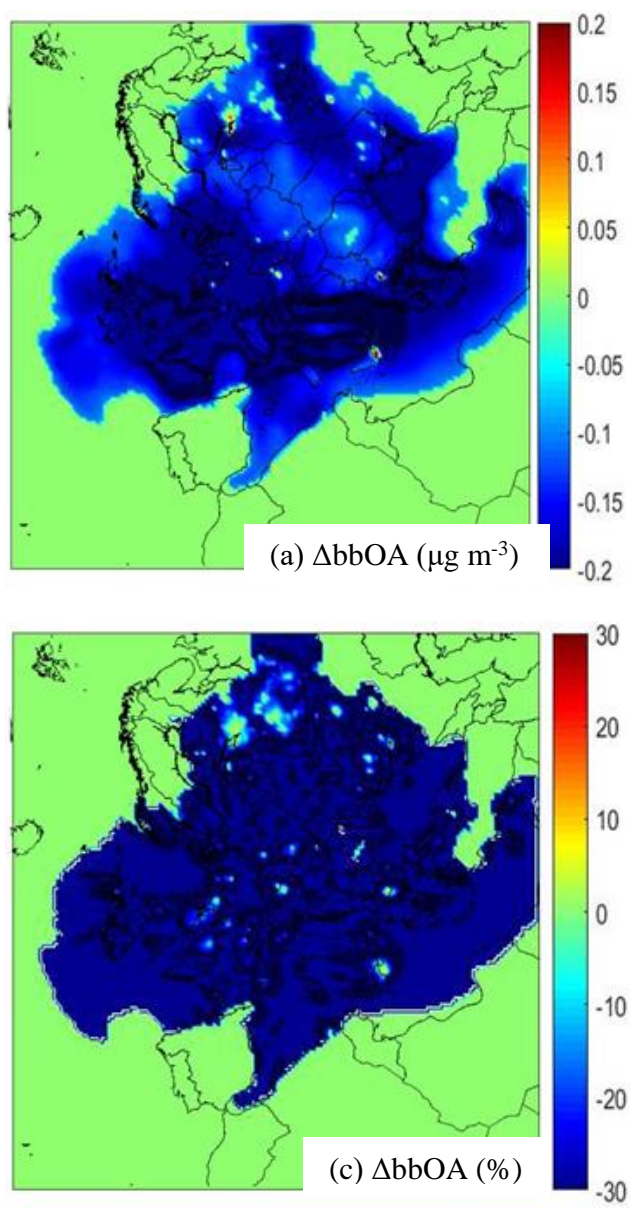

Winter
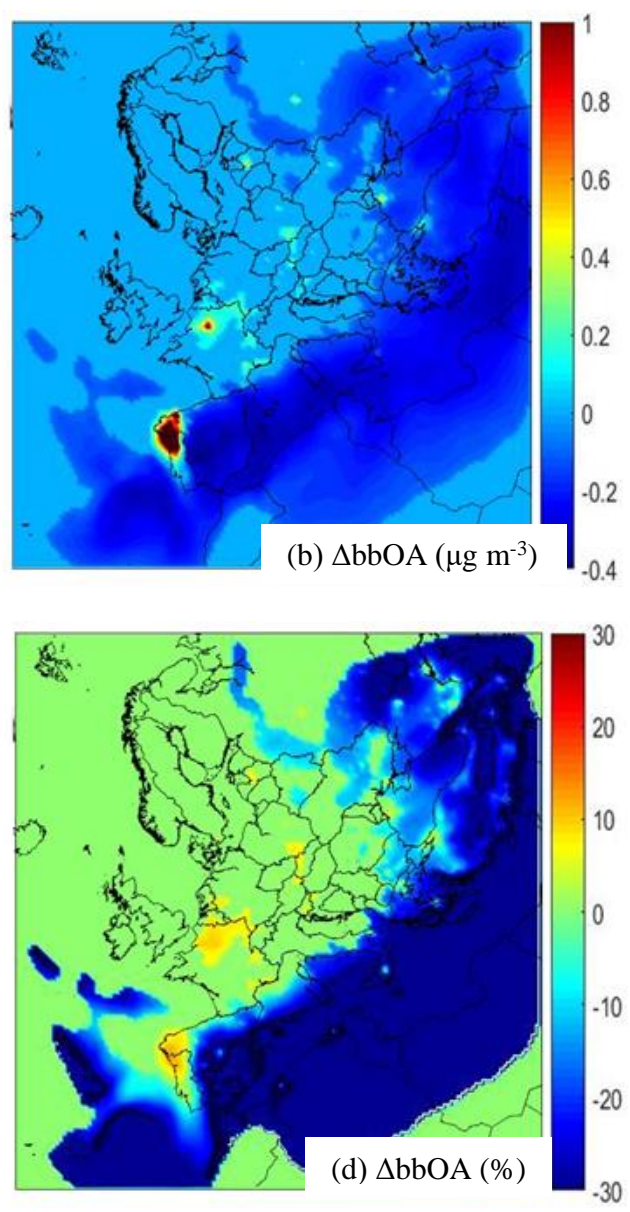

5

6 Figure S4. Average predicted absolute $\left(\mu \mathrm{g} \mathrm{m}^{-3}\right)$ change of ground-level $\mathrm{PM}_{2.5}$ total

7 bbOA concentrations from PMCAMx-SR base case and sensitivity simulations during

8 the modeled summer and winter periods (a-b). Also shown the corresponding relative

9 (\%) change of ground-level $\mathrm{PM}_{2.5}$ total bbOA concentrations during the modeled 10 periods (c-d). Positive values indicate that PMCAMx-SR sensitivity run predicts 11 higher concentrations. 